Hier steht eine Anzeige.

\section{Springer}

\title{
PD-1-Blocker bei Melanompatienten mit Autoimmunerkrankung oder irAE?
}

Anti-PD-1-Antikörper sind klinisch aktiv bei zahlreichen Tumorerkrankungen. In alle bisherigen Studien mit diesen Checkpointinhibitoren wurden nur Patienten ohne signifkante vorbestehende Autoimmunerkrankungen (AD) aufgenommen.

$\mathrm{N}$ ach Kenntnisstand der Wissenschaftler gab es nur eine Studie, an der auch wenige Patienten mit schwerwiegenden immunbezogenen unerwünschten Ereignissen (irAE) nach Behandlung mit dem CTLA-4-Hemmer Ipilimumab teilnahmen. Eigene Ergebnisse von ihnen legen nahe, dass Ipilimumab auch bei Patienten mit AD bedeutende antitumorale Aktivität hat.
AD-Flare (Tab. 1) und bedurften einer immunsuppressiven Behandlung. Patienten mit gastrointestinalen oder neurologischen Krankheitsbildern blieben von Flares verschont, und nur insgesamt 2 Patienten beendeten die Behandlung Flare-bedingt. Die Ansprechrate von Patienten mit irAE erreichte $40 \%$. Im gesamten Patientenkollektiv kam es zu keinem Therapie-assoziierten Todesfall.

\begin{tabular}{l|l}
$\begin{array}{l}\text { Tab. 1: Toxizität der PD-1-Hemmer bei Patienten mit Autoimmunerkrankung } \\
\text { Reaktion }\end{array}$ & Patientenzahl (\%) (n= 52) \\
\hline $\begin{array}{l}\text { Aufflammen der AD unter PD-1-Hemmung } \\
\text { Nein }\end{array}$ & $32(62 \%)$ \\
\hline Ja & $20(38 \%)$ \\
\hline Grad des Aufflammens & \\
\hline G1-2 & $17(33 \%)$ \\
\hline G3 & $3(6 \%)$ \\
\hline G4 & 0 \\
\hline Tage bis zum Aufflammen (range) & median 38 (8-161) \\
\hline
\end{tabular}

Anti-PD-1-Antikörper erwiesen sich als effektiver und weniger toxisch als Ipilimumab. Bisher existierten keine oder nur spärliche Daten zur Sicherheit und Wirksamkeit einer PD-1-Inhibition bei Krebspatienten mit vorbestehender AD oder signifikanten Toxizitäten nach Ipilimumab-Therapie.

Ihre retrospektive Analyse stützten die Forscher auf die Krankenblätter von 119 Patienten, die zwischen 2012 und 2015 in 13 internationalen MelanomZentren trotz AD und/oder irAE nach Ipilimumab-Therapie PD-1-Hemmer erhalten hatten.

$33 \%$ der Patienten mit vorbestehender AD sprachen auf die Therapie mit dem Checkpointhemmer an. 20 erlitten einen
Fazit: Die PD-1-Inhibition zur Behandlung des fortgeschrittenen Melanoms induziert bei Patienten mit vorbestehenden $\mathrm{AD}$ oder irAE infolge einer Ipilimumab-Behandlung vermehrt immunologische Toxizitäten. Diese verlaufen aber oft mild, sind leicht beherrschbar und führen selten zum vorzeitigen Therapieabbruch. Die Forscher sehen darin die Bestätigung der Sicherheit und des klinischen Nutzens einer Anti-PD1-Therapie auch für solche Melanompatienten.

Wolfgang Zimmermann

Menzies AM et al. Anti-PD-1 therapy in patients with advanced melanoma and preexisting autoimmune disorders or major toxicity with ipilimumab. Ann Oncol. 2017;28(2):368-76. 